\title{
Packing of spanning mixed arborescences
}

\author{
Hui Gao \\ Center for Discrete Mathematics \\ Fuzhou University \\ Fuzhou, Fujian 350108, China \\ E-mail: gaoh1118@yeah.net
}

\author{
Daqing Yang* \\ Department of Mathematics \\ Zhejiang Normal University \\ Jinhua, Zhejiang 321004, China \\ E-mail: dyang@zjnu.edu.cn
}

May 8, 2020

\begin{abstract}
In this paper, we characterize a mixed graph $F$ which contains $k$ edge and arc disjoint spanning mixed arborescences $F_{1}, \ldots, F_{k}$, such that for each $v \in V(F)$, the cardinality of $\left\{i \in[k]: v\right.$ is the root of $\left.F_{i}\right\}$ lies in some prescribed interval. This generalizes both Nash-Williams and Tutte's theorem on spanning tree packing for undirected graphs and the previous characterization on digraphs which was given by Cai [in: Arc-disjoint arborescences of digraphs, J. Graph Theory 7(2) (1983), 235-240] and Frank [in: On disjoint trees and arborescences, Algebraic Methods in Graph Theory, Colloquia Mathematica Soc. J. Bolyai, Vol. 25 (North-Holland, Amsterdam) (1978), 159-169].
\end{abstract}

Keywords: Tree; Arborescence; Packing; Digraph; Mixed graph AMS subject classifications. 05B35, 05C40, 05C70

\section{Introduction}

In this paper, we consider graphs which may have multiple edges or (and) arcs but not loops. A mixed graph $F=(V ; E, A)$ is a graph consisting of the set $E$ of undirected edges and the set $A$ of directed arcs. Let $X_{1}, \ldots, X_{t}$ be disjoint subsets of $V$, we call $\mathcal{P}=\left\{X_{1}, \ldots, X_{t}\right\}$ a subpartition of $V$ and particularly a partition of $V$ if $V=\cup_{j=1}^{t} X_{j}$. Denote $e_{E}(\mathcal{P})=\mid\left\{e \in E\right.$ : one end of $e$ belongs to some $X_{i}$ and the other end belongs to another $X_{j}$ with $j \neq i$ or $\left.V \backslash \cup_{j=1}^{t} X_{j}\right\} \mid$. Denote the set $\{1, \ldots, k\}$ by $[k]$. For a function $f: V \rightarrow \mathbb{N}$, define a set function $\tilde{f}: 2^{V} \rightarrow \mathbb{N}$ as $\widetilde{f}(X)=\sum_{x \in X} f(x)$, where $X \subseteq V$.

Nash-Williams [13] and Tutte [14] independently characterized when an undirected graph has $k$ edge-disjoint spanning trees.

\footnotetext{
*Corresponding author, grant number: NSFC 11871439.
} 
Theorem 1.1 ([13, 14]) For a graph $G=(V, E)$, there exist $k$ edge disjoint spanning trees, if and only if for any partition $\mathcal{P}=\left\{X_{0}, X_{1}, \ldots, X_{t}\right\}$ of $V$,

$$
e_{E}(\mathcal{P}) \geq k t
$$

Let $D=(V, A)$ be a digraph. A subdigraph of $D$ is spanning if its vertex set is $V$. A subdigraph $F$ (it may not be spanning) of $D$ is an $r$-arborescence if its underlying graph is a tree and for any $u \in V(F)$, there is exactly one directed path in $F$ from $r$ to $u$, vertex $r$ is the root of arborescence $F$.

As a directed version of Theorem 1.1, Edmonds' theorem [4] characterizes directed graphs that contain $k$ arc disjoint spanning arborescences with prescribed roots in terms of a cut condition.

Theorem 1.2 ([4]) For a digraph $D=(V, A)$, let $R=\left\{r_{1}, \ldots, r_{k}\right\} \subseteq V$ be a multiset. For $i=1, \ldots, k$, there exist arc-disjoint spanning $r_{i}$-arborescneces in $D$, if and only if for any $\emptyset \neq X \subseteq V$,

$$
d_{A}^{-}(X) \geq\left|\left\{r_{i}: r_{i} \notin X\right\}\right|
$$

Throughout this paper, $F=(V ; E, A)$ is a mixed graph, $R=\left\{r_{1}, \ldots, r_{k}\right\} \subseteq V$ is a multiset. By regarding each undirected edge as a directed arc in both directions, each concept in directed graphs can be naturally extended to mixed graphs. Especially, a subdigraph $P$ of $F$ is a mixed path if its underlying graph is a path and one end of $P$ can be reached from the other. A subdigraph $T$ (it may not be spanning) of $F$ is called an $r$-mixed arborescence if its underlying graph is a tree and for any $u \in V(T)$, there is exactly one mixed path in $T$ from $r$ to $u$. Equivalently, a subgraph $T$ of $F$ is an $r$-mixed arborescence if there exists an orientation of the undirected edges of $T$ such that the obtained subgraph (whose arc set is the union of original arc set and oriented arc set of $T)$ is an $r$-arborescence.

The following result is due to Frank [6], it generalized Theorem 1.1 and Theorem [1.2 to mixed graphs when $r_{1}=r_{2}=\cdots=r_{k}$. Since mixed graphs may contain edges and arcs, a property that holds for mixed graphs should hold for both directed and undirected graphs.

Theorem $1.3([6])$ Let $F=(V ; E, A)$ be a mixed graph, $r \in V$, and $k$ a positive integer. There exist $k$ edge and arc disjoint spanning $r$-mixed arborescences in $F$, if and only if, for any subpartition $\left\{X_{1}, \ldots, X_{t}\right\}$ of $V-r$,

$$
e_{E}(\mathcal{P})+\sum_{j=1}^{t} d_{A}^{-}\left(X_{j}\right) \geq k t .
$$

For nonempty $X, Z \subseteq V$, let $Z \rightarrow X$ denote that $X$ and $Z$ are disjoint and $X$ is reachable from $Z$, that is, there is a mixed path from $Z$ to $X$. We shall write $v$ for $\{v\}$ for simplicity. Let $P(X):=X \cup\{v \in V \backslash X: v \rightarrow X\}$. 
Let $D$ and $R$ be as in Theorem 1.2. The following remarkable extension of Edmonds' theorem (by Kamiyama, Katoh and Takizawa [10]) enables us to find a packing of reachable arborescences in digraph $D$ for $R$, that is packing arc disjoint $r_{i}$-arborescences $F_{i}$ in $D$ for $1 \leq i \leq k$ such that $V\left(F_{i}\right)=\left\{v \in V: v\right.$ is reachable from $r_{i}$ in $\left.D\right\}$.

Theorem 1.4 ([10]) In a digraph $D=(V, A)$, let $R=\left\{r_{1}, \ldots, r_{k}\right\} \subseteq V$ be a multiset. There exists a packing of reachable arborescences in digraph $D$ for $R$ if and only if for any $\emptyset \neq X \subseteq V$,

$$
d_{A}^{-}(X) \geq\left|\left\{r_{i}: r_{i} \in P(X) \backslash X\right\}\right|
$$

For packing of reachable arborescences, further extensions have been made, such as its mixed version [12, matroidal version [11, and matroidal mixed version [9]. Some other developments for packing of arborescences in the recent years include matroidbased packing [3], and its hypergraphic version [5], under cardinality constraints [8]. Refer [5] for more details.

In this paper, we are interested in the following extension of Edmonds' theorem, which is due to Cai [2] and Frank [6] (see also Theorem 10.1.11 in Frank [7]). This extension characterized a digraph $D$ which contains $k$ arc disjoint spanning arborescences $F_{1}, \ldots, F_{k}$, such that for each $v \in V(D)$, the cardinality of $\left\{i \in[k]: v\right.$ is the root of $\left.F_{i}\right\}$ lies in some prescribed interval.

Theorem $1.5([\mathbf{2}, 6])$ Let $D=(V, A)$ be a digraph, $f, g: V \rightarrow \mathbb{N}$ be functions such that $f \leq g$. Then there exist $k$ arc-disjoint spanning arborescences $F_{1}, \ldots, F_{k}$ in $D$ for which $F_{i}$ is rooted at some $r_{i} \in V$ for $1 \leq i \leq k$ such that $f(v) \leq\left|\left\{i \in[k]: r_{i}=v\right\}\right| \leq g(v)$ for $v \in V$, if and only if,

(i) $\tilde{f}(V) \leq k$;

(ii) for any subpartition $\left\{X_{1}, \ldots, X_{t}\right\}$ of $V$,

$$
\sum_{j=1}^{t} d_{A}^{-}\left(X_{j}\right) \geq k(t-1)+\tilde{f}\left(V \backslash \cup_{j=1}^{t} X_{j}\right)
$$

(iii) for any $\emptyset \neq X \subseteq V$,

$$
d_{A}^{-}(X) \geq k-\widetilde{g}(X)
$$

In this paper, we generalize Theorem 1.1 and Theorem 1.5 to mixed graphs, which is the following.

Theorem 1.6 Let $F=(V ; E, A)$ be a mixed graph, $f, g: V \rightarrow \mathbb{N}$ be functions such that $f \leq g$. Then there exist $k$ edge and arc disjoint spanning mixed arborescences $F_{1}, \ldots, F_{k}$ in $F$ for which $F_{i}$ is rooted at some $r_{i} \in V$ for $1 \leq i \leq k$ and $f(v) \leq \mid\left\{i \in[k]: r_{i}=\right.$ $v\} \mid \leq g(v)$ for $v \in V$, if and only if, 
(i) $\tilde{f}(V) \leq k$;

(ii) for any subpartition $\mathcal{P}=\left\{X_{1}, \ldots, X_{t}\right\}$ of $V$,

$$
e_{E}(\mathcal{P})+\sum_{j=1}^{t} d_{A}^{-}\left(X_{j}\right) \geq k(t-1)+\tilde{f}\left(V \backslash \cup_{j=1}^{t} X_{j}\right)
$$

(iii) for any subpartition $\mathcal{P}=\left\{X_{1}, \ldots, X_{t}\right\}$ of $V$,

$$
e_{E}(\mathcal{P})+\sum_{j=1}^{t} d_{A}^{-}\left(X_{j}\right) \geq k t-\widetilde{g}\left(\cup_{j=1}^{t} X_{j}\right)
$$

For the proof of our result, besides what have been used by Nash-Williams [13], Tutte [14, Cai [2] and Frank [6], we adopt a technique named as properly intersecting elimination operation (PIEO for simplicity), which was first introduced by Bérczi and Frank [1] (to the best of our knowledge), studied and used again by Gao and Yang [8]. Indeed, we shall use some similar approaches to [8] in our proofs of Theorem 1.6.

\section{Proof of Theorem 1.6}

We shall use some definitions and propositions that have been presented in [8].

Let $\Omega$ be a finite set. Two subsets $X, Y \subseteq \Omega$ are intersecting if $X \cap Y \neq \emptyset$ and properly intersecting if $X \cap Y, X \backslash Y$, and $Y \backslash X \neq \emptyset$. A function $p: 2^{\Omega} \rightarrow \mathbb{Z}$ is supermodular (intersecting supermodular), where $2^{\Omega}$ denotes the power set of $\Omega$, if the inequality

$$
p(X)+p(Y) \leq p(X \cup Y)+p(X \cap Y)
$$

holds for all subsets (intersecting subsets, respectively) of $\Omega$. A function $b$ is submodular if $-b$ is supermodular.

Let $\mathcal{F}$ be a multiset, which consists of some subsets of $\Omega$ (these subsets do not have to be different). Let $\cup \mathcal{F}$ be the union of elements in $\mathcal{F}$ (then $\cup \mathcal{F} \subseteq \Omega$ ). Let $x \in \Omega$ and $\mathcal{F}(x)$ denote the number of elements in $\mathcal{F}$ containing $x$. If there exist no properly intersecting pairs in $\mathcal{F}$, then $\mathcal{F}$ is laminar. If there exists a properly intersecting pair $X$ and $Y$ in $\mathcal{F}$, then we obtain $\mathcal{F}^{\prime}$ from $\mathcal{F}$ by replacing $X$ and $Y$ with one of the following three types of subset(s):

Type 1, $X \cup Y$ and $X \cap Y$, denoted as $\mathcal{F} \stackrel{1}{\rightarrow} \mathcal{F}^{\prime}$;

Type 2, $X \cup Y$, denoted as $\mathcal{F} \stackrel{2}{\rightarrow} \mathcal{F}^{\prime}$;

Type $3, X \cap Y$, denoted as $\mathcal{F} \stackrel{3}{\rightarrow} \mathcal{F}^{\prime}$.

A properly intersecting elimination operation (PIEO for simplicity) on $X$ and $Y$ in $\mathcal{F}$ is defined to be one of the above three types.

Let $Z_{1}$ and $Z_{2}$ be multisets. Denote by $Z_{1} \uplus Z_{2}$ the multiset union of $Z_{1}$ and $Z_{2}$, that is, for any $z$, the number of $z$ in $Z_{1} \uplus Z_{2}$ is the total number of $z$ in $Z_{1}$ and $Z_{2}$. 
Let $\mathcal{D}(\Omega)$ be the set that consists of all families of disjoint subsets of $\Omega$. From now on, we suppose $\mathcal{F}_{1}, \mathcal{F}_{2} \in \mathcal{D}(\Omega)$. We adopt PIEOs in $\mathcal{G}_{0}=\mathcal{F}_{1} \uplus \mathcal{F}_{2}$, step by step, and obtain families $\mathcal{G}_{0}, \ldots, \mathcal{G}_{i-1}, \mathcal{G}_{i}, \ldots$ of subsets of $\Omega$. In [8], it has already been proved that the process of PIEOs will terminate. Suppose the obtained families of subsets of $\Omega$ are $\mathcal{G}_{0}, \ldots, \mathcal{G}_{n}$. Then $\mathcal{G}_{n}$ is laminar. For $X, Y \in \mathcal{G}_{i}$, if $X \subseteq Y$, then define $X \leq Y$. Let $\mathcal{G}_{i}^{\prime}$ be the family of maximal elements in $\mathcal{G}_{i}, \mathcal{F}_{3}:=\mathcal{G}_{n}^{\prime}$ and $\mathcal{F}_{4}:=\mathcal{G}_{n} \backslash \mathcal{F}_{3}$.

Proposition 2.1 ([8, Proposition 3.2]) If $X, Y \in \mathcal{G}_{i}$ are properly intersecting, then $X, Y \in \mathcal{G}_{i}^{\prime}$.

Proposition 2.2 ([8, Proposition 3.4]) The following hold true:

(i) $\mathcal{F}_{3}, \mathcal{F}_{4} \in \mathcal{D}(\Omega) . \cup \mathcal{F}_{4} \subseteq\left(\cup \mathcal{F}_{1}\right) \cap\left(\cup \mathcal{F}_{2}\right)$.

(ii) Moreover, $\cup \mathcal{F}_{4}=\left(\cup \mathcal{F}_{1}\right) \cap\left(\cup \mathcal{F}_{2}\right)$ if and only if for any $i \in[n], \mathcal{G}_{i-1} \stackrel{1}{\rightarrow} \mathcal{G}_{i}$.

Now we are ready for the proof of Theorem 1.6.

$(\Rightarrow)$ Necessity: Suppose there exist $k$ edge and arc disjoint spanning mixed arborescences $F_{1}, \ldots, F_{k}$ in $F$ for which $F_{i}$ is rooted at some $r_{i} \in V$ for $1 \leq i \leq k$ and $f(v) \leq\left|\left\{i \in[k]: r_{i}=v\right\}\right| \leq g(v)$ for $v \in V$,

Then there exists an oriented arc set $A^{\prime}$ of $E$, for which there exist $k$ arc-disjoint spanning arborescences $F_{1}^{\prime}, \ldots, F_{k}^{\prime}$ in $D=\left(V, A \cup A^{\prime}\right)$ such that $F_{i}^{\prime}$ is rooted at $r_{i}$ for $1 \leq i \leq k$ and $f(v) \leq\left|\left\{i \in[k]: r_{i}=v\right\}\right| \leq g(v)$ for $v \in V$. Obviously, $\widetilde{f}(V) \leq k$. By Theorem 1.5, (11) and (2) hold in $D$. Let $\mathcal{P}=\left\{X_{0}, X_{1}, \ldots, X_{t}\right\}$ be a partition of $V$. Since $A^{\prime}$ is an oriented arc set of $E, e_{E}(\mathcal{P}) \geq \sum_{j=1}^{t} d_{A^{\prime}}^{-}\left(X_{j}\right)$. Hence, by (1) ,

$$
e_{E}(\mathcal{P})+\sum_{j=1}^{t} d_{A}^{-}\left(X_{j}\right) \geq \sum_{j=1}^{t} d_{A^{\prime}}^{-}\left(X_{j}\right)+\sum_{j=1}^{t} d_{A}^{-}\left(X_{j}\right)=\sum_{j=1}^{t} d_{A \cup A^{\prime}}^{-}\left(X_{j}\right) \geq k(t-1)+\widetilde{f}\left(X_{0}\right),
$$

this is (3). By (2),

$$
e_{E}(\mathcal{P})+\sum_{j=1}^{t} d_{A}^{-}\left(X_{j}\right) \geq \sum_{j=1}^{t} d_{A \cup A^{\prime}}^{-}\left(X_{j}\right) \geq \sum_{j=1}^{t}\left(k-\widetilde{g}\left(X_{j}\right)\right)=k t-\widetilde{g}\left(\cup_{j=1}^{t} X_{j}\right),
$$

this is (4).

$(\Leftarrow)$ Sufficiency: We prove the sufficiency by induction on $|E|$. For the base step, suppose $E=\emptyset$; and then apply Theorem 1.5 ,

For the induction step, suppose $E \neq \emptyset$. We shall prove that we can orient an edge $e \in E$ to $\vec{e}$, such that after we do $A:=A+\vec{e}, E:=E-e, F^{\prime}:=(V ; A, E)$, assumptions (3) and (44) still hold for the new mixed graph $F^{\prime}$. Then by the induction hypothesis, there exist $k$ edge and arc disjoint mixed arborescences $F_{1}, \ldots, F_{k}$ in $F^{\prime}$ for which $F_{i}$ is rooted at some $r_{i} \in V$ for $1 \leq i \leq k$ such that $f(v) \leq\left|\left\{i \in[k]: r_{i}=v\right\}\right| \leq g(v)$ for 
$v \in V$. If $\vec{e} \notin \cup_{i=1}^{k} F_{i}$, then $F$ includes $F_{1}, \ldots, F_{k}$ as demanded; if $\vec{e} \in E\left(F_{i_{0}}\right)$ for some $i_{0} \in[k]$, then $F$ includes $F_{1}, \ldots, F_{i_{0}}-\vec{e}+e, \ldots, F_{k}$ as demanded.

The critical point that determines an orientation of $e$ lies on the subpartitions of $V$ that make assumptions (3) or (4) tight in $F$. These critical subpartitions are defined next; $\mathcal{E}^{1}$ is aimed at (3), $\mathcal{E}^{2}$ is aimed at (44). This explains why the subpartitions in $\mathcal{E}^{1}$ and $\mathcal{E}^{2}$ will play some central roles next.

Define

$$
\begin{aligned}
& \mathcal{E}^{1}:=\left\{\mathcal{F} \in \mathcal{D}(V): e_{E}(\mathcal{F})+\sum_{X \in \mathcal{F}} d_{A}^{-}(X)=k(t-1)+\widetilde{f}(V \backslash \cup \mathcal{F})\right\} ; \\
& \mathcal{E}^{2}:=\left\{\mathcal{F} \in \mathcal{D}(V): e_{E}(\mathcal{F})+\sum_{X \in \mathcal{F}} d_{A}^{-}(X)=k t-\widetilde{g}(\cup \mathcal{F})\right\} .
\end{aligned}
$$

Suppose $\mathcal{F}_{1}, \mathcal{F}_{2} \in \mathcal{E}^{1} \cup \mathcal{E}^{2}$, denote

$$
E\left(\mathcal{F}_{1}, \mathcal{F}_{2}\right):=\left\{e \in E \text { : one end of } e \text { is in } \cup \mathcal{F}_{1} \backslash \cup \mathcal{F}_{2} \text { and the other is in } \cup \mathcal{F}_{2} \backslash \cup \mathcal{F}_{1}\right\} \text {. }
$$

Process of PIEOs. Let $\mathcal{G}_{0}=\mathcal{F}_{1} \uplus \mathcal{F}_{2}$. We adopt PIEOs of Type 1 in $\mathcal{G}_{0}=\mathcal{F}_{1} \uplus \mathcal{F}_{2}$, step by step, and obtain families $\mathcal{G}_{0}, \ldots, \mathcal{G}_{n}$ of subsets of $V$; equivalently, for $0 \leq i \leq n-1$, we replace some properly intersecting pair $X$ and $Y$ in $\mathcal{G}_{i-1}$ with $X \cup Y$ and $X \cap Y$, and obtain $\mathcal{G}_{i}$. Recall that $\mathcal{G}_{i}^{\prime}$ is the family of maximal elements in $\mathcal{G}_{i}, \mathcal{F}_{3}:=\mathcal{G}_{n}^{\prime}$ and $\mathcal{F}_{4}:=\mathcal{G}_{n} \backslash \mathcal{F}_{3}$. By Proposition $2.2, \mathcal{F}_{3}, \mathcal{F}_{4} \in \mathcal{D}(V)$.

\section{Claim $2.3 \quad$ (i) $\left|\mathcal{F}_{1}\right|+\left|\mathcal{F}_{2}\right|=\left|\mathcal{F}_{3}\right|+\left|\mathcal{F}_{4}\right|$.}

(ii) $\cup \mathcal{F}_{3}=\left(\cup \mathcal{F}_{1}\right) \cup\left(\cup \mathcal{F}_{2}\right)$.

Proof. For $i \in[n]$, suppose we replace a properly intersecting pair $X$ and $Y$ in $\mathcal{G}_{i-1}$ with $X \cup Y$ and $X \cap Y$, and obtain $\mathcal{G}_{i}$. Clearly, $\left|\mathcal{G}_{i-1}\right|=\left|\mathcal{G}_{i}\right|$. It follows that $\left|\mathcal{F}_{1}\right|+\left|\mathcal{F}_{2}\right|=$ $\left|\mathcal{G}_{0}\right|=\left|\mathcal{G}_{n}\right|=\left|\mathcal{F}_{3}\right|+\left|\mathcal{F}_{4}\right|$.

By Proposition 2.1, $X, Y \in \mathcal{G}_{i-1}^{\prime}$, and thus $X \cup Y \in \mathcal{G}_{i}^{\prime}$. So $\mathcal{G}_{i}^{\prime}$ consists of $X \cup Y$ and all the subsets in $\mathcal{G}_{i-1}^{\prime}$ not contained in $X \cup Y$; this proves $\cup \mathcal{G}_{i-1}^{\prime}=\cup \mathcal{G}_{i}^{\prime}$. Hence $\cup \mathcal{G}_{0}^{\prime}=\cup \mathcal{G}_{n}^{\prime}$.

By definition, $\left(\cup \mathcal{F}_{1}\right) \cup\left(\cup \mathcal{F}_{2}\right)=\cup \mathcal{G}_{0}^{\prime}$; since $\cup \mathcal{G}_{0}^{\prime}=\cup \mathcal{G}_{n}^{\prime}$ and $\mathcal{G}_{n}^{\prime}=\mathcal{F}_{3}$, we have $\left(\cup \mathcal{F}_{1}\right) \cup\left(\cup \mathcal{F}_{2}\right)=\cup \mathcal{G}_{0}^{\prime}=\cup \mathcal{G}_{n}^{\prime}=\cup \mathcal{F}_{3}$.

Claim 2.4 For $\mathcal{F}_{1}, \mathcal{F}_{2} \in \mathcal{E}^{1} \cup \mathcal{E}^{2}$, we have

$$
\begin{gathered}
e_{E}\left(\mathcal{F}_{1}\right)+\sum_{X \in \mathcal{F}_{1}} d_{A}^{-}(X)+e_{E}\left(\mathcal{F}_{2}\right)+\sum_{X \in \mathcal{F}_{2}} d_{A}^{-}(X) \\
\geq e_{E}\left(\mathcal{F}_{3}\right)+\sum_{X \in \mathcal{F}_{3}} d_{A}^{-}(X)+e_{E}\left(\mathcal{F}_{4}\right)+\sum_{X \in \mathcal{F}_{4}} d_{A}^{-}(X)+\left|E\left(\mathcal{F}_{1}, \mathcal{F}_{2}\right)\right| .
\end{gathered}
$$

Proof. Define $A^{\prime \prime}$ is an orientation of $E$ as following: 
PACKING OF SPANNING MiXED ARBORESCENCES

- if $e=u v \in E$ satisfied that $u \notin \cup \mathcal{F}_{1}$ and $v \in \cup \mathcal{F}_{1}$, orient $e$ from $u$ to $v$ in $A^{\prime \prime}$;

- else if $e=u v \in E$ satisfied that $u \notin \cup \mathcal{F}_{2}$ and $v \in \cup \mathcal{F}_{2}$, orient $e$ from $u$ to $v$ in $A^{\prime \prime}$;

- else, orient the rest of $E$ arbitrarily in $A^{\prime \prime}$.

Then $e_{E}\left(\mathcal{F}_{1}\right)=\sum_{X \in \mathcal{F}_{1}} d_{A^{\prime \prime}}^{-}(X)$, and $e_{E}\left(\mathcal{F}_{2}\right)=\sum_{X \in \mathcal{F}_{2}} d_{A^{\prime \prime}}^{-}(X)+\left|E\left(\mathcal{F}_{1}, \mathcal{F}_{2}\right)\right|$. Hence,

$$
\begin{aligned}
& e_{E}\left(\mathcal{F}_{1}\right)+\sum_{X \in \mathcal{F}_{1}} d_{A}^{-}(X)+e_{E}\left(\mathcal{F}_{2}\right)+\sum_{X \in \mathcal{F}_{2}} d_{A}^{-}(X) \\
= & \sum_{X \in \mathcal{F}_{1}} d_{A^{\prime \prime}}^{-}(X)+\sum_{X \in \mathcal{F}_{1}} d_{A}^{-}(X)+\sum_{X \in \mathcal{F}_{2}} d_{A^{\prime \prime}}^{-}(X)+\left|E\left(\mathcal{F}_{1}, \mathcal{F}_{2}\right)\right|+\sum_{X \in \mathcal{F}_{2}} d_{A}^{-}(X) \\
= & \sum_{X \in \mathcal{F}_{1}} d_{A \cup A^{\prime \prime}}^{-}(X)+\sum_{X \in \mathcal{F}_{2}} d_{A \cup A^{\prime \prime}}^{-}(X)+\left|E\left(\mathcal{F}_{1}, \mathcal{F}_{2}\right)\right| \\
= & \sum_{X \in \mathcal{G}_{0}} d_{A \cup A^{\prime \prime}}^{-}(X)+\left|E\left(\mathcal{F}_{1}, \mathcal{F}_{2}\right)\right|
\end{aligned}
$$

By Claim 2.3 $(i i), \cup \mathcal{F}_{3}=\left(\cup \mathcal{F}_{1}\right) \cup\left(\cup \mathcal{F}_{2}\right)$. For every $e=u v \in E$ such that $u \notin$ $\left(\cup \mathcal{F}_{1}\right) \cup\left(\cup \mathcal{F}_{2}\right)$ and $v \in\left(\cup \mathcal{F}_{1}\right) \cup\left(\cup \mathcal{F}_{2}\right)$, by the definition of $A^{\prime \prime}$, $e$ is oriented from $u$ to $v$. Therefore $e_{E}\left(\mathcal{F}_{3}\right)=\sum_{X \in \mathcal{F}_{3}} d_{A^{\prime \prime}}^{-}(X)$.

By Proposition 2.2, $\cup \mathcal{F}_{4}=\left(\cup \mathcal{F}_{1}\right) \cap\left(\cup \mathcal{F}_{2}\right)$. For every $e=u v \in E$ such that $u \notin$ $\left(\cup \mathcal{F}_{1}\right) \cap\left(\cup \mathcal{F}_{2}\right)$ and $v \in\left(\cup \mathcal{F}_{1}\right) \cap\left(\cup \mathcal{F}_{2}\right)$, by the definition of $A^{\prime \prime}$, $e$ is oriented from $u$ to $v$. Therefore $e_{E}\left(\mathcal{F}_{4}\right)=\sum_{X \in \mathcal{F}_{4}} d_{A^{\prime \prime}}^{-}(X)$. Hence,

$$
\begin{aligned}
& e_{E}\left(\mathcal{F}_{3}\right)+\sum_{X \in \mathcal{F}_{3}} d_{A}^{-}(X)+e_{E}\left(\mathcal{F}_{4}\right)+\sum_{X \in \mathcal{F}_{4}} d_{A}^{-}(X) \\
= & \sum_{X \in \mathcal{F}_{3}} d_{A^{\prime \prime}}^{-}(X)+\sum_{X \in \mathcal{F}_{3}} d_{A}^{-}(X)+\sum_{X \in \mathcal{F}_{4}} d_{A^{\prime \prime}}^{-}(X)+\sum_{X \in \mathcal{F}_{4}} d_{A}^{-}(X) \\
= & \sum_{X \in \mathcal{F}_{3}} d_{A \cup A^{\prime \prime}}^{-}(X)+\sum_{X \in \mathcal{F}_{4}} d_{A \cup A^{\prime \prime}}^{-}(X) \\
= & \sum_{X \in \mathcal{G}_{n}} d_{A \cup A^{\prime \prime}}^{-}(X) \quad\left(\text { since } \mathcal{G}_{n}=\mathcal{F}_{3} \uplus \mathcal{F}_{4}\right) .
\end{aligned}
$$

In the process of PIEOs, for $i \in[n]$, suppose we obtain $\mathcal{G}_{i}$ by replacing a properly intersecting pair $X$ and $Y$ in $\mathcal{G}_{i-1}$ with $X \cup Y$ and $X \cap Y$. Then $\mathcal{G}_{i-1} \backslash\{X, Y\}=$ $\mathcal{G}_{i} \backslash\{X \cup Y, X \cap Y\}$. Since $d_{A \cup A^{\prime \prime}}^{-}$is submodular on $2^{V}, d_{A \cup A^{\prime \prime}}^{-}(X)+d_{A \cup A^{\prime \prime}}^{-}(Y) \geq d_{A \cup A^{\prime \prime}}^{-}(X \cup$ $Y)+d_{A \cup A^{\prime \prime}}^{-}(X \cap Y)$. Therefore $\sum_{X \in \mathcal{G}_{i-1}} d_{A \cup A^{\prime \prime}}^{-}(X) \geq \sum_{X \in \mathcal{G}_{i}} d_{A \cup A^{\prime \prime}}^{-}(X)$. It follows that

$$
\sum_{X \in \mathcal{G}_{0}} d_{A \cup A^{\prime \prime}}^{-}(X) \geq \sum_{X \in \mathcal{G}_{1}} d_{A \cup A^{\prime \prime}}^{-}(X) \geq \ldots \geq \sum_{X \in \mathcal{G}_{n}} d_{A \cup A^{\prime \prime}}^{-}(X) .
$$


Hence, we have

$$
\begin{aligned}
& e_{E}\left(\mathcal{F}_{1}\right)+\sum_{X \in \mathcal{F}_{1}} d_{A}^{-}(X)+e_{E}\left(\mathcal{F}_{2}\right)+\sum_{X \in \mathcal{F}_{2}} d_{A}^{-}(X) \\
& =\sum_{X \in \mathcal{G}_{0}} d_{A \cup A^{\prime \prime}}^{-}(X)+\left|E\left(\mathcal{F}_{1}, \mathcal{F}_{2}\right)\right| \quad \text { (by (15)) } \\
& \geq \sum_{X \in \mathcal{G}_{n}} d_{A \cup A^{\prime \prime}}^{-}(X)+\left|E\left(\mathcal{F}_{1}, \mathcal{F}_{2}\right)\right| \quad \text { (by (17)) } \\
& =e_{E}\left(\mathcal{F}_{3}\right)+\sum_{X \in \mathcal{F}_{3}} d_{A}^{-}(X)+e_{E}\left(\mathcal{F}_{4}\right)+\sum_{X \in \mathcal{F}_{4}} d_{A}^{-}(X)+\left|E\left(\mathcal{F}_{1}, \mathcal{F}_{2}\right)\right| \quad(\text { by (6) }) \text {. }
\end{aligned}
$$

The following lemma will be used in the final step to explain why we can orient an edge $e \in E$ to take care of all these critical subpartitions in $\mathcal{E}^{1}$ and $\mathcal{E}^{2}$.

Lemma 2.5 For $\mathcal{F}_{1}, \mathcal{F}_{2} \in \mathcal{E}^{1} \cup \mathcal{E}^{2}$, we have $E\left(\mathcal{F}_{1}, \mathcal{F}_{2}\right)=\emptyset$.

Proof. Suppose to the contrary that $E\left(\mathcal{F}_{1}, \mathcal{F}_{2}\right) \neq \emptyset$. Then, by Claim 2.4, we have

$$
\begin{aligned}
e_{E}\left(\mathcal{F}_{1}\right)+\sum_{X \in \mathcal{F}_{1}} d_{A}^{-}(X)+e_{E}\left(\mathcal{F}_{2}\right)+\sum_{X \in \mathcal{F}_{2}} d_{A}^{-}(X) \\
>e_{E}\left(\mathcal{F}_{3}\right)+\sum_{X \in \mathcal{F}_{3}} d_{A}^{-}(X)+e_{E}\left(\mathcal{F}_{4}\right)+\sum_{X \in \mathcal{F}_{4}} d_{A}^{-}(X) .
\end{aligned}
$$

Case 1: Assume $\mathcal{F}_{1}, \mathcal{F}_{2} \in \mathcal{E}^{1}$.

By Claim $2.3(i i), V \backslash \cup \mathcal{F}_{3}=\left(V \backslash \cup \mathcal{F}_{1}\right) \cap\left(V \backslash \cup \mathcal{F}_{2}\right)$. By Proposition 2.2, $V \backslash \cup \mathcal{F}_{4}$ $=\left(V \backslash \cup \mathcal{F}_{1}\right) \cup\left(V \backslash \cup \mathcal{F}_{2}\right)$. Thus,

$$
\tilde{f}\left(V \backslash \cup \mathcal{F}_{3}\right)+\widetilde{f}\left(V \backslash \cup \mathcal{F}_{4}\right)=\tilde{f}\left(V \backslash \cup \mathcal{F}_{1}\right)+\widetilde{f}\left(V \backslash \cup \mathcal{F}_{2}\right) .
$$

Hence,

$$
\begin{aligned}
& e_{E}\left(\mathcal{F}_{3}\right)+\sum_{X \in \mathcal{F}_{3}} d_{A}^{-}(X)+e_{E}\left(\mathcal{F}_{4}\right)+\sum_{X \in \mathcal{F}_{4}} d_{A}^{-}(X) \\
& \geq k\left(\left|\mathcal{F}_{3}\right|-1\right)+\tilde{f}\left(V \backslash \cup \mathcal{F}_{3}\right)+k\left(\left|\mathcal{F}_{4}\right|-1\right)+\widetilde{f}\left(V \backslash \cup \mathcal{F}_{4}\right) \quad(\text { by }(3)) \\
& \left.=k\left(\left|\mathcal{F}_{1}\right|-1\right)+\widetilde{f}\left(V \backslash \cup \mathcal{F}_{1}\right)+k\left(\left|\mathcal{F}_{2}\right|-1\right)+\widetilde{f}\left(V \backslash \cup \mathcal{F}_{2}\right) \quad \text { (by (91) and Claim (2.3) }(i)\right) \\
& =e_{E}\left(\mathcal{F}_{1}\right)+\sum_{X \in \mathcal{F}_{1}} d_{A}^{-}(X)+e_{E}\left(\mathcal{F}_{2}\right)+\sum_{X \in \mathcal{F}_{2}} d_{A}^{-}(X) \quad\left(\text { since } \mathcal{F}_{1}, \mathcal{F}_{2} \in \mathcal{E}^{1}\right),
\end{aligned}
$$

but this is a contradiction to (8) .

Case 2: Assume $\mathcal{F}_{1}, \mathcal{F}_{2} \in \mathcal{E}^{2}$. 
The proof will use the function $g$ and the assumption (4), also the definition of $\mathcal{E}^{2}$. The process is similar to Case 1, details are skipped here.

Case 3: Assume $\mathcal{F}_{1} \in \mathcal{E}^{1}$ and $\mathcal{F}_{2} \in \mathcal{E}^{2}$.

By Claim 2.3 (ii), $V \backslash \cup \mathcal{F}_{3} \subseteq V \backslash \cup \mathcal{F}_{1}$, and $\left(V \backslash \cup \mathcal{F}_{1}\right) \backslash\left(V \backslash \cup \mathcal{F}_{3}\right)=\cup \mathcal{F}_{3} \backslash \cup \mathcal{F}_{1}=$ $\left(\left(\cup \mathcal{F}_{1}\right) \cup\left(\cup \mathcal{F}_{2}\right)\right) \backslash \cup \mathcal{F}_{1}=\cup \mathcal{F}_{2} \backslash \cup \mathcal{F}_{1}$. By Proposition $\left[2.2, \cup \mathcal{F}_{4} \subseteq \cup \mathcal{F}_{2}\right.$, and $\cup \mathcal{F}_{2} \backslash \cup \mathcal{F}_{4}=$ $\cup \mathcal{F}_{2} \backslash\left(\left(\cup \mathcal{F}_{1}\right) \cap\left(\cup \mathcal{F}_{2}\right)\right)=\cup \mathcal{F}_{2} \backslash \cup \mathcal{F}_{1}$. Thus $\left(V \backslash \cup \mathcal{F}_{1}\right) \backslash\left(V \backslash \cup \mathcal{F}_{3}\right)=\cup \mathcal{F}_{2} \backslash \cup \mathcal{F}_{4}$.

By the assumption $f \leq g$, using $V \backslash \cup \mathcal{F}_{3} \subseteq V \backslash \cup \mathcal{F}_{1}$ and $\cup \mathcal{F}_{4} \subseteq \cup \mathcal{F}_{2}$, we have

$$
\begin{aligned}
& \tilde{f}\left(V \backslash \cup \mathcal{F}_{1}\right)-\tilde{f}\left(V \backslash \cup \mathcal{F}_{3}\right)=\tilde{f}\left(\left(V \backslash \cup \mathcal{F}_{1}\right) \backslash\left(V \backslash \cup \mathcal{F}_{3}\right)\right) \\
\leq & \tilde{g}\left(\left(V \backslash \cup \mathcal{F}_{1}\right) \backslash\left(V \backslash \cup \mathcal{F}_{3}\right)\right)=\tilde{g}\left(\cup \mathcal{F}_{2} \backslash \cup \mathcal{F}_{4}\right)=\tilde{g}\left(\cup \mathcal{F}_{2}\right)-\widetilde{g}\left(\cup \mathcal{F}_{4}\right) .
\end{aligned}
$$

Hence,

$$
\begin{array}{rlr} 
& e_{E}\left(\mathcal{F}_{3}\right)+\sum_{X \in \mathcal{F}_{3}} d_{A}^{-}(X)+e_{E}\left(\mathcal{F}_{4}\right)+\sum_{X \in \mathcal{F}_{4}} d_{A}^{-}(X) & \\
\geq & k\left(\left|\mathcal{F}_{3}\right|-1\right)+\widetilde{f}\left(V \backslash \cup \mathcal{F}_{3}\right)+k\left|\mathcal{F}_{4}\right|-\widetilde{g}\left(\cup \mathcal{F}_{4}\right) & \text { (by (33) and (41)) } \\
\geq & k\left(\left|\mathcal{F}_{1}\right|-1\right)+\tilde{f}\left(V \backslash \cup \mathcal{F}_{1}\right)+k\left|\mathcal{F}_{2}\right|-\widetilde{g}\left(\cup \mathcal{F}_{2}\right) & \text { (by (10) and Claim 2.3 (i)) } \\
= & e_{E}\left(\mathcal{F}_{1}\right)+\sum_{X \in \mathcal{F}_{1}} d_{A}^{-}(X)+e_{E}\left(\mathcal{F}_{2}\right)+\sum_{X \in \mathcal{F}_{2}} d_{A}^{-}(X) & \text { (since } \left.\mathcal{F}_{1} \in \mathcal{E}^{1}, \mathcal{F}_{2} \in \mathcal{E}^{2}\right),
\end{array}
$$

but this is a contradiction to (18). This proves the lemma.

To finish the proof, we pick an edge $e_{0} \in E$, orient $e_{0}$ to $\vec{e}_{0}$ as following: If there exists an $\mathcal{F}_{0} \in \mathcal{E}^{1} \cup \mathcal{E}^{2}$ such that one end of $e_{0}$, say $v \in \cup \mathcal{F}_{0}$ and the other end $u \notin \cup \mathcal{F}_{0}$, then we orient $e_{0}$ from $u$ to $v$ (i.e., $\vec{e}_{0}:=\overrightarrow{u v}$ ); otherwise, orient $e_{0}$ to $\vec{e}_{0}$ arbitrarily. Then we do $A:=A+\vec{e}_{0}, E:=E-e_{0}, F^{\prime}:=(V ; A, E)$. It suffices for us to prove that for any $\mathcal{F} \in \mathcal{D}(V)$, assumptions (3) and (4) still hold for this new mixed graph $F^{\prime}$.

Note that the subpartitions in $\mathcal{E}^{1}$ and $\mathcal{E}^{2}$ are the ones that make assumptions (3) or (44) tight in the mixed graph $F$. If $\mathcal{F} \notin \mathcal{E}^{1} \cup \mathcal{E}^{2}$, since $e_{E}(\mathcal{F})+\sum_{X \in \mathcal{F}} d_{A}^{-}(X)$ is decreased by at most 1, (3) and (44) still hold in $F^{\prime}$. Otherwise, we have $\mathcal{F} \in \mathcal{E}^{1} \cup \mathcal{E}^{2}$. Then we prove (next) that $e_{E}(\mathcal{F})+\sum_{X \in \mathcal{F}} d_{A}^{-}(X)$ keeps the same in $F$ and $F^{\prime}$. Thus (3) and (4) still hold in $F^{\prime}$.

Suppose $\vec{e}_{0}=\overrightarrow{u v}$ in $F^{\prime}$. If both $u, v \in X$ for some $X \in \mathcal{F}$, or both $u, v \notin \cup \mathcal{F}$, then $e_{E}(\mathcal{F})$ and $\sum_{X \in \mathcal{F}} d_{A}^{-}(X)$ keep the same in $F$ and $F^{\prime}$.

If, for some $X, Y \in \mathcal{F}$ and $X \neq Y, v \in X$ and $u \in Y$, then $e_{E}(\mathcal{F})$ is decreased by 1 and $\sum_{X \in \mathcal{F}} d_{A}^{-}(X)$ is increased by 1 in $F^{\prime}$. Therefore $e_{E}(\mathcal{F})+\sum_{X \in \mathcal{F}} d_{A}^{-}(X)$ keeps the same in $F$ and $F^{\prime}$.

The left cases are either $(\mathrm{A}) v \in \cup \mathcal{F}$ and $u \notin \cup \mathcal{F}$; or $(\mathrm{B}) u \in \cup \mathcal{F}$ and $v \notin \cup \mathcal{F}$. But Case (B) can not happen. Indeed, assume to the contrary that $u \in \cup \mathcal{F}$ and $v \notin \cup \mathcal{F}$. Since $\vec{e}_{0}$ is oriented from $u$ to $v$, there exists an $\mathcal{F}_{0} \in \mathcal{E}^{1} \cup \mathcal{E}^{2}$ such that $v \in \cup \mathcal{F}_{0}$ and $u \notin \cup \mathcal{F}_{0}$. we conclude that $e_{0} \in E\left(\mathcal{F}_{0}, \mathcal{F}\right)$. But by Lemma 2.5, $E\left(\mathcal{F}_{0}, \mathcal{F}\right)=\emptyset$. This is a contradiction. So the only left case is $(\mathrm{A}) v \in \cup \mathcal{F}$ and $u \notin \cup \mathcal{F}$. Then $e_{E}(\mathcal{F})$ is decreased by 1 and $\sum_{X \in \mathcal{F}} d_{A}^{-}(X)$ is increased by 1 in $F^{\prime}$. Therefore $e_{E}(\mathcal{F})+\sum_{X \in \mathcal{F}} d_{A}^{-}(X)$ keeps the same in $F$ and $F^{\prime}$. This finishes the proof of Theorem 1.6 . 
PACKIng of SPANNING MiXed ARBORESCENCES

\section{References}

[1] K. Bérczi, A. Frank, Supermodularity in unweighted graph optimization I: Branchings and matchings, Math. Oper. Res. 43(3) (2018), 726-753.

[2] M.-C. Cai, Arc-disjoint arborescences of digraphs, J. Graph Theory 7(2) (1983), 235-240.

[3] O. Durand de Gevigney, V.-H. Nguyen, and Z. Szigeti, Matroid-based packing of arborescences, SIAM J. Discrete Math., 27 (2013), 567-574.

[4] J. Edmonds, Edge-disjoint branchings, Combinatorial algorithms (Courant Comput. Sci. Sympos. 9, New York Univ., New York, 1972), pp. 91-96. Algorithmics Press, New York, 1973.

[5] Q. Fortier, Cs. Király, M. Léonard, Z. Szigeti, A. Talon, Old and new results on packing arborescences in directed hypergraphs, Discrete Appl. Math. 242 (2018), 26-33.

[6] A. Frank, On disjoint trees and arborescences, Algebraic Methods in Graph Theory, Colloquia Mathematica Soc. J. Bolyai, Vol. 25 (North-Holland, Amsterdam) (1978), 159-169.

[7] A. Frank, Connections in Combinatorial Optimization, Oxford Lecture Series in Mathematics and Its Applications, Vol. 38 (Oxford University Press, Oxford, UK) (2011).

[8] H. Gao, D. Yang, Packing branchings under cardinality constraints on their root sets, arXiv:1908.10795v2 [math.CO] 9 Feb 2020 (to appear in European J. Combin.).

[9] H. Gao, D. Yang, Packing of maximal independent mixed arborescences, arXiv:2003.04062 1 [math.CO] 9 Mar 2020.

[10] N. Kamiyama, N. Katoh, and A. Takizawa, Arc-disjoint in-trees in directed graphs, Combinatorica, 29 (2009), 197-214.

[11] Cs. Király, On maximal independent arborescence packing, SIAM J. Discrete. Math. 30 (4) (2016), 2107-2114.

[12] T. Matsuoka, S. Tanigawa, On reachability mixed arborescence packing, Discrete Optimization 32 (2019), 1-10.

[13] C. St. J. A. Nash-Williams, Edge-disjoint spanning trees of finite graphs. J. Lond. Math. Soc. 36 (1961), 445-450.

[14] W. T. Tutte, On the problem of decomposing a graph into $n$ connected factors. J. Lond. Math. Soc. 36 (1961), 221-230. 